\title{
Rapid prototyping of three-dimensional biomodels as an adjuvant in the surgical planning for intracranial aneurysms ${ }^{1}$
}

\author{
Bruna Olandoski ErbanoI, Ana Cristina Opolski', Marcia Olandoski", José Aguiomar Foggiatto ${ }^{\mathrm{III}}$, Luiz Fernando Kubrusly ${ }^{\mathrm{IV}}$, \\ Ulrich Andreas Dietz ${ }^{\mathrm{V}}$, Cassio Zini ${ }^{\mathrm{VI}}$, Melissa Mitsue Makita Arantes Marinho ${ }^{\mathrm{VI}}$, André Giacomelli Leal ${ }^{\mathrm{VII}}$, Ricardo Ramina ${ }^{\mathrm{VIII}}$ \\ ${ }^{\mathrm{I}}$ Graduate student, School of Medicine, Evangelic Faculty of Parana, Curitiba-PR, Brazil. Acquisition, analysis and interpretation of data, manuscript \\ writing. \\ "PhD, Biostatistics Division, Pontifical Catholic University of Parana, Curitiba-PR, Brazil. Critical revision. \\ IIIPhD, Rapid Prototyping Division, Federal University of Technology, Curitiba-PR, Brazil. Scientific and intellectual content of the study, critical \\ revision.

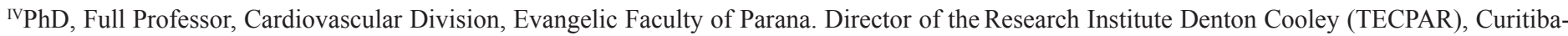 \\ PR, Brazil. Critical revision, final approval of the version to be published. \\ vPhD, Full Professor, Pediatric Division, Evangelic Faculty of Parana, Curitiba-PR, Brazil. Critical revision, final approval of the version to be \\ published.

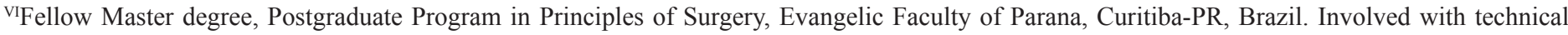 \\ procedures. \\ ${ }^{\mathrm{VII}} \mathrm{MSc}$, Vascular and Endovascular Neurosurgery Division, Neurology Institute of Curitiba, Curitiba-PR, Brazil. Conception, design, intellectual and \\ scientific content of the study, critical revision, final approval of the version to be published. \\ VIIIPhD, Head of Neurosurgery Division, Neurology Institute of Curitiba, Curitiba-PR, Brazil. Critical revision.
}

\section{ABSTRACT}

PURPOSE: To fabricate a three-dimensional biomodels of intracranial aneurysms, using rapid prototyping technology, to facilitate optimal anatomical visualization of aneurysms prior to and during surgery.

METHODS: Four intracranial aneurysms cases were selected for this study. Using CT angiography images, the rapid prototyping process was completed using a PolyJet technology machine. The size and morphology of the prototypes were compared to brain digital subtraction arteriography of the same patients.

RESULTS: The biomodels reproduced the exact location and morphology of the intracranial aneurysms, particularly the necks, in lifesize dimensions and exactly the same as measured by digital subtraction arteriography. The arterial segments adjacent to the aneurysm and arteries anatomically known by the surgeon were also shown, which could guide the surgeon to the aneurysmal segment. The models showed an average unit cost of US\$ 130 and each one took an average of 20 hours to be fabricated.

CONCLUSIONS: It is possible to fabricate 3D physical biomodels of intracranial aneurysms from CT angiography images. These prototypes may be useful in the surgical planning for intracranial aneurysms to clarify the anatomy, define surgical techniques and facilitate the choice of suitable materials, such as clips and clip appliers.

Key words: Models, Anatomic. Intracranial Aneurysm

\section{Introduction}


It is estimated that non-ruptured intracranial aneurysms (IA) affect 0.6 to $6 \%$ of the population ${ }^{1}$. The risk of rupture is approximately $2 \%$ per year ${ }^{2}$, which represents the primary complication of IA, and is responsible for $80 \%$ of all spontaneous subarachnoid hemorrhage cases $^{3}$. The mortality risk for it is approximately $60 \%$ at six months ${ }^{1}$, which substantiates the importance of appropriate IA treatment.

The surgical treatment of saccular IA consists of exclusion of the aneurysmal sac from the cerebral circulation through microsurgical clipping or endovascular embolization ${ }^{4}$. To facilitate treatment, as well as assist in choosing the surgical technique and strategy, various diagnostic methods are employed. Among the non-invasive diagnostic methods, CT angiography (CTA) is the most commonly used because it achieves nearly $100 \%$ specificity and $95 \%$ sensitivity for the evaluation of polygon Willis aneurysms ${ }^{5}$. However, for presenting structures in twodimensional images, CTA can limit visualization of the aneurysmal neck $^{6}$, which is the main region that needs to be clearly identified by the surgeon during pre-operative procedures ${ }^{7}$.

The main difficulties during the treatment of IA include predicting appropriate materials to be used, anatomical variations and procedural complications. It is well known, particularly in complex and wide-neck saccular aneurysms, that surgical planning is vital ${ }^{8}$ to avoid excessive manipulation of the intracranial vessels and an extended surgical time, which are predisposing factors for aneurysmal rupture, infection and increased morbidity and mortality rates ${ }^{9}$.

Rapid prototyping (RP) comprises a set of technologies and automated processes capable of generating objects in three dimensions (3D), from two-dimensional images. It is an orderly process of overlapping layers of liquid resin; thus, it is possible to manufacture $3 \mathrm{D}$ structures in an automated and inexpensive manner ${ }^{10}$. In the healthcare field, the use of RP technology has been demonstrated to be suitable for fabricating maxillary prosthetics ${ }^{11}$ and cranioplasty molds ${ }^{12}$. In the field of vascular surgery, studies have reported the use of 3D models in surgical planning for aortic aneurysms ${ }^{13}$. The application of RP to IA has been suggested ${ }^{14-16}$, but less explored.

Thus, the aim of this study is to establish the feasibility of fabricating 3D biomodels of saccular IA and to demonstrate their use as an adjuvant in surgical planning.

\section{Methods}

The project was approved by the Ethics Committee of the Evangelical School of Paraná, which included waived informed consent, and followed the statements of Helsinki Declaration and 196/96 Resolution of the Brazilian National Council of Health.

The study was conducted in two phases. The first consisted of establishing the methodology to manufacture the pilot-prototype. The second described the initial experience with clinical cases, based on the same technique used in the first phase. It was fabricated three additional prototypes of IA of three different patients.

\section{First phase}

The fabrication of the pilot-prototype began by acquiring a CTA brain image containing a saccular aneurysm, which came from the database of the Neurology Institute of Curitiba. The CTA image was composed of axial helical slices, coded in the international standard format called Digital Imaging and Communications in Medicine. Next, the file was transferred to the Center for Rapid Prototyping and Tooling Technology of the Federal Technological University of Paraná, where the entire process was developed.

The next step was creation of three-dimensionality: the file in Digital Imaging and Communications in Medicine format was segmented and transformed into a stereolithography file format, which converts the image on a triangular mesh. The software used included InVesalius, which is in the public domain, and Mimics $^{\circledR}$ (Materialise's Interactive Medical Image Control System). These software systems are capable of defining a specific window of density, which enable differentiation of structures based on density, for example, bones from soft tissue. The nonionic iodinated contrast media (Iopamiron ${ }^{\circledR}$ ) used in CTA imaging makes vascular structures denser than the surrounding tissue, so the software is able to uniquely identify the angioarchitecture. The software also allows isolation of the segment of the structures of interest, in this case the vessel containing the aneurysm, as well as some adjacent arteries.

Next, it was necessary to create a surface possible to be printed, a 3D angiogram. The triangular mesh of the stereolithography format was edited with CATIA $^{\circledR}$, a computer aided design program, to remove potential artifacts.

Once the three-dimensional computerized model was obtained, an impression was made using the Objet $^{\circledR}$ Eden 250 Rapid Prototyping machine. The Objet Studio software, on this machine, promotes a sequence of slices in the $3 \mathrm{D}$ model, whose 
distance is constant, to obtain geometric information of each layer separately. The printing is performed by overlapping these separate layers. After deposition of a resin layer, an elevator contained inside the machine lowers the entire prototype, so that the next layer is deposited 16 microns above the previous one. Finally, the prototype materialized by sequential overlapping of 2D layers.

The biomodel was fabricated out of a photosensitive liquid resin called FullCure 720. This resin solidifies when exposed to ultraviolet light, allowing the overlapping layer process. Given the structural complexity of the biomodel and to ensure integrity, another resin, FullCure 705, served as the support. After the Rapid Prototyping process had finished, the support material was removed manually. The FullCure 720 resin has the advantage of the ability to be sterilized by ethylene oxide, enabling the manipulation of the prototype during surgery.

\section{Second phase}

Following the methodology employed in the manufacture of the pilot-prototype, three other models of IA of three different patients were printed to determinate if the prototypes are capable of reproducing the anatomy of saccular IA regardless of their location. These models had IA in the most commonly found locations in the circle of Willis: anterior communicating artery $(35 \%)$, posterior communicating artery $(35 \%)$ and middle cerebral artery bifurcation $(20 \%)^{17}$. Subsequently, the morphology and dimensions of the prototypes were compared to brain digital subtraction arteriography images, considered to be the gold standard for IA visualization ${ }^{3}$, previously performed in these same patients.

\section{Results}

\section{First phase}

After completion of the first phase, a 3D resin model was obtained which reproduced the exact location of an IA in life-size dimensions and therefore established the feasibility of the methodology. This model contained an aneurysm of the right middle cerebral artery of $4 \times 4 \times 3 \mathrm{~mm}$, used 16 grams of resin to manufacture and took one hour and 24 minutes to be prototyped. The entire process cost US\$100 and took four days to be completed, from acquisition of the CTA through final materialized prototype.

\section{Second phase}

In the second phase, three prototyped biomodels were fabricated which reproduced the exact location of the three IA, in the most commonly found IA locations in the circle of Willis ${ }^{17}$. The biomodels were printed in life-size so that the IA morphology, especially the aneurysmal neck, to be found by the surgeon during the surgical treatment, could be predicted with greater accuracy. For each biomodel it took an average of 20 hours to complete, from the acquisition of the CTA images through final materialized biomodel.

The first biomodel of this stage revealed a bilobed aneurysm of the anterior communicating artery, measuring $10 \times 5$ x $5 \mathrm{~mm}$. This biomodel used 29 grams of resin and took two hours and 49 minutes to be prototyped with a total cost of US\$ 170 .

The second biomodel showed two aneurysms, one in the anterior communicating artery, measuring $4 \times 4 \times 3 \mathrm{~mm}$, and another at the bifurcation of the right middle cerebral artery, measuring $5 \times 4 \times 2.5 \mathrm{~mm}$. This biomodel used 24 grams of resin and took $125 \mathrm{~min}$ to be prototyped, with a total cost of US\$135.

The third prototype contained a right posterior communicating artery aneurysm, measuring $2.1 \times 2.2 \times 3 \mathrm{~mm}$, which used 25 grams of resin and took 93 minutes to be prototyped, with a total cost of US\$ 110 .

The biomodels were compared to digital subtraction arteriography of each patient. All three prototypes represented an accurate prediction of the aneurysm angioarchitecture, especially the neck, with exact the same dimensions as measured by arteriography. Also, the biomodel accurately represented arterial segments adjacent to the aneurysm and arteries anatomically known by the surgeon, which could serve as a guide to the aneurysmal segment (Figures 1-4).

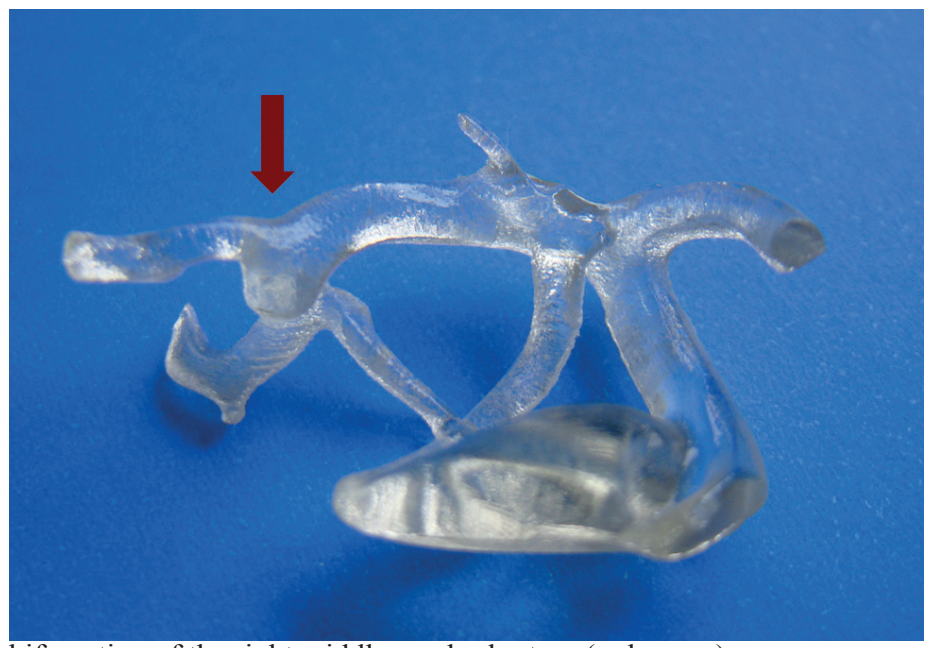

bifurcation of the right middle cerebral artery (red arrow). 

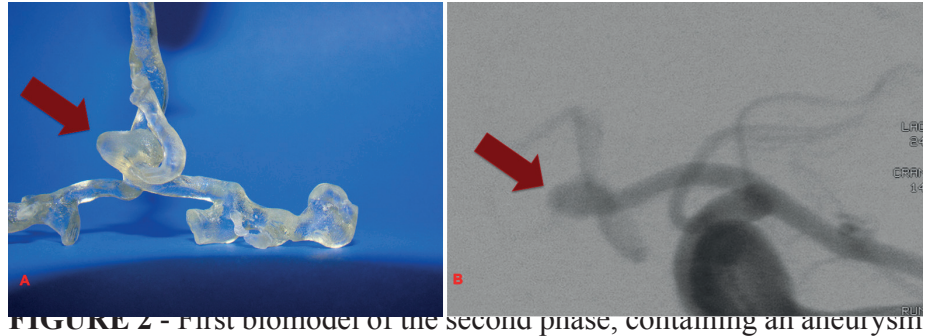

in the anterior communicating artery (red arrow) (A). Brain digital subtraction arteriography of the same patient (B).

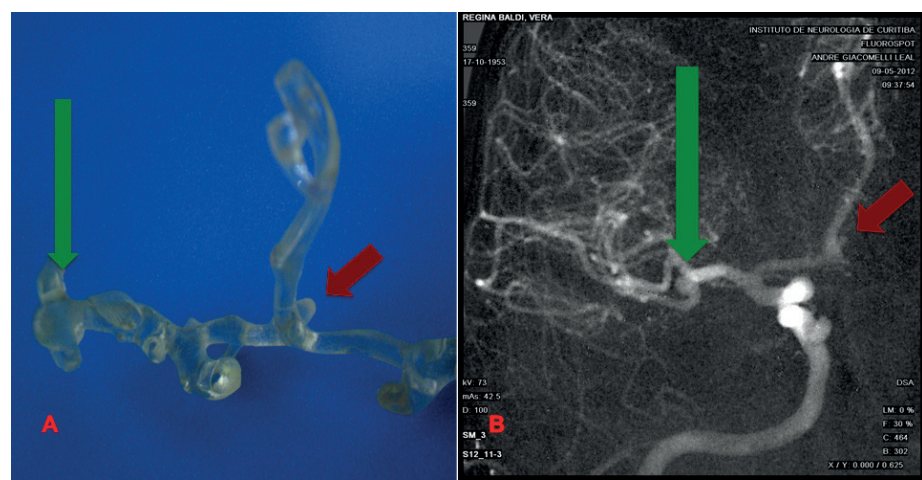

FIGURE 3 - Second biomodel of the second phase, containing two intracranial aneurysms; one in the right middle cerebral artery (green arrow) and another in the anterior communicating artery (red arrow) (A). Brain digital subtraction arteriography of the same patient (B).
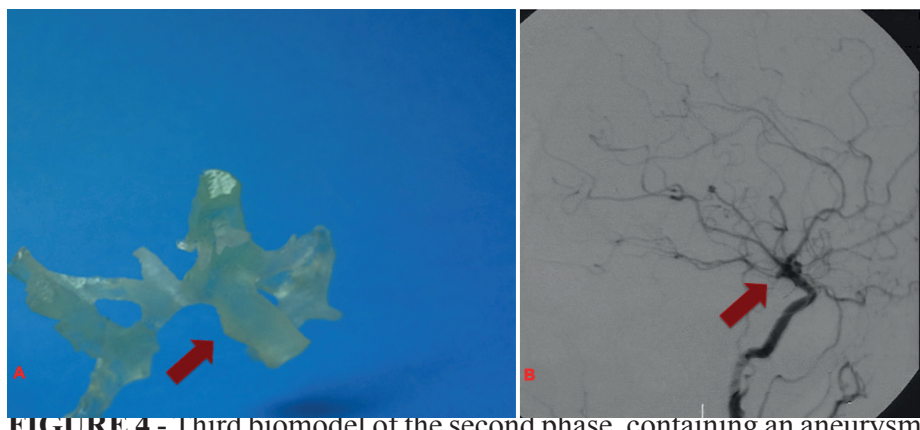
of the posterior communicating artery (red arrow) (A). Brain digital subtraction arteriography of the same patient $(\mathbf{B})$.

\section{Discussion}

This study showed the feasibility of manufacturing 3D IA biomodels using rapid prototyping technology, from CTA images, to be used as an adjuvant in surgical planning. In 1990, Mankovich et al. ${ }^{18}$ reported the first replication of human anatomical structures and, since then, RP has been widely used in many areas of healthcare. The most similarly related application of it is in making maxillary prosthetics ${ }^{11}$, cranioplasty molds ${ }^{12}$ and for surgical planning of aortic aneurysms ${ }^{13}$. In the vascular neurosurgery field, in 1999, D’Urso et al. ${ }^{16}$ first described the use of RP technique to fabricate IA prototypes, and reported that biomodels could optimize IA's treatment.
In the case of surgical techniques, studies suggested that biomodels may assist in the choice between an endovascular and an open surgical approach, as well as in the choice of the best surgical access to be used in microsurgery clipping cases ${ }^{15}$. Both microsurgery clipping and embolization procedures for IA involve many technical details. It is commonly difficult to predict the size, quantity and angles of the clips to be used for each aneurysm. Thus, being identical to the actual structures, biomodels can facilitate the prediction of surgical techniques and approaches during the preoperative period $^{14,15}$.

Emphasis is placed, especially in cases of complex anatomy, on the ability to view the anatomical structures and its surroundings, from any perspective, to provide a better understanding of the angioarchitecture of the aneurysm and arteries involved in each case ${ }^{16}$. Generally, patients with complex wide neck aneurysms have an indication for microsurgical clipping $^{19}$, which demands greater skill of the surgeon performing the procedure ${ }^{15}$. Less experienced surgeons, residents or even those who have not attained the learning curve, may benefit from biomodels, both for anatomical recognition ${ }^{16}$ and for handling the clips and appliers ${ }^{15,20}$. While currently the amount of open surgery is limited, the use of RP for surgical simulations of IA could be crucial to enhance the skills of less experienced surgeons.

At the beginning, Wurm et al. ${ }^{14}$ believed that the vessel wall, the geometry of the neck, the configuration and direction of the aneurysm and evidence of small perforating arteries would be the most important characteristics targeted by the surgeon. After application of a questionnaire to five surgeons, this hypothesis was confirmed.

Previously studies showed that the main disadvantages of using RP for surgical planning were a delay of the process and associated costs. Kimura et al. ${ }^{15}$ reported that seven days are required to prototype a biomodel and the associated cost to do it is around US\$ 300 to 400 . In this study, the average cost of each biomodel was US\$ 130 and, once the methodology was determined in the pilot-prototype phase, biomodels required an average of 20 hours to be done from acquisition of CTA through final materialized biomodel. The time taken here was half of that reported by Wurm et al. ${ }^{14}$ and $1 / 8$ of the time reported by Kimura et al. ${ }^{15}$.

For emergency cases, such as ruptured aneurysms, a time of 20 hours is inappropriate ${ }^{15}$. However, 20 hours is acceptable for elective surgeries, considering that surgical and preoperative planning take a few days. Moreover, the cost of producing a biomodel, compared to the total cost of the surgery, becomes insignificant. 
The process of informed consent to the patient about the diagnosis of IA and its treatment, with possible risks and benefits, is another significant advantage of this technique. As a matter of fact, biomodels can facilitate the explanation of the case and surgical risk to the patients and their relatives ${ }^{14,15}$. Studies have reported that the existence of a physical prototype, in fact, assisted in obtaining informed consent of the patients, improved understanding the disease and the surgical procedure that would be performed ${ }^{14,16}$. Finally, it is important to emphasize that an actual physical model of the 3D surgical anatomy preoperatively provides great confidence to the surgeon, and consequently to the patient, and may even reduce the surgical time and risk.

One of the limitations found in this process was the unfeasibility of making hollow prototypes resembling the vascular structure as much as possible. Moreover, prototypes do not show the presence of intra-arterial thrombus or calcification, which has previously been described ${ }^{14,16}$. However, according to Kimura et al..$^{15}$, although there are advantages to making hollow prototypes, the thickness and texture of the material would not be representative of the real vascular system.

With respect to methodology, first, although other studies have demonstrated the possibility of using different imaging methods as the main data source for making prototypes from angioressonance ${ }^{16}$ and $3 \mathrm{D}$ angiograms ${ }^{14}$, this process favors the use of CTA, as it is minimally invasive and already part of the preoperative IA surgical routine. Second, the CTA, once resolved in Digital Imaging and Communications in Medicine format, is three-dimensionally reconstructed as a mesh of multiple triangles, which must be edited to avoid the formation of artifacts. This process is operator-dependent and requires deep anatomy knowledge to avoid modifications to the morphology of the structures. Third, a challenging part of the process is determination of the window density that can isolate only the structures of interest. The intracranial vessels, when impregnated by contrast, become denser and resemble bone structures, thereby differing from the brain parenchyma. Thus, if the chosen window density is too high, the images show artifacts. If the window density is too low, the image loses details of smaller vessels.

\section{Conclusions}

It is possible to fabricate a three-dimensional identical physical biomodels of IA from CTA images. These prototypes are representative of the IA and, since they predict aneurysmal architecture, may better define the surgical technique and the appropriate choice of materials.

\section{References}

1. Steiner T, Juvela S, Unterberg A, Jung C, Forsting M, Rinkel G. European Stroke Organization Guidelines for the management of intracranial aneurysms and subarachnoid haemorrhage. Cerebrovasc Dis. 2013;35(2):93-112

2. Rinkel GJ, Djibuti M, Algra A, van Gijn J. Prevalence and risk of rupture of intracranial aneurysms: a systematic review. Stroke. 1998;29(1):251-6.

3. Suarez JI, Tarr RW, Selman WR. Aneurysmal subarachnoid hemorrhage. N Engl J Med. 2006;354(4):387-96.

4. Li H, Pan R, Wang H, Rong X, Yin Z, Milgrom DP, Shi X, Tang Y, Peng Y. Clipping versus coiling for ruptured intracranial aneurysms: a systematic review and meta-analysis. Stroke. 2013;44(1):29-37.

5. Andrade GC, Teixeira PAD, Alves HFP, Donato EE, Moreira CAA, Faria RCS, Dias LAA. Diagnóstico dos aneurismas cerebrais por angiotomografia tridimensional. Arq Neuropsiquiatr. 2003;61(1):748.

6. Yasui T, Kishi H, Komiyama M, Iwai Y, Yamanaka K, Nishikawa M, Nakajima H, Morikawa T. The limitations of three-dimensional CT angiography (3D-CTA) in the diagnosis of cerebral aneurysms. No Shinkei Geka. 2000;28(11):975-81.

7. Guglielmi G, Vinuela F, Duckwiler G, Dion J, Lylyk P, Berenstein A, Strother C, Graves V, Halbach V, Nichols D, Hopkins N, Ferguson R, Sepetka I. Endovascular treatment of posterior circulation aneurysms by electrothrombosis using electrically detachable coils. J Neurosurg. 1992;77(4):515-24.

8. Lawton MT, Spetzler RF. Surgical management of giant intracranial aneurysms: experience with 171 patients. Clin Neurosurg. $1995 ; 42: 245-66$

9. Rinne J, Hernesniemi J, Niskanen M, Vapalahti M. Management outcome for multiple intracranial aneurysms. Neurosurgery. 1995;36(1):31-7.

10. Tukuru N, Gowda S, Ahmed SM, Badami S. Rapid prototype technique in medical field. Res J Pharm Tech. 2008;1(4):341-4.

11. Winder J, Bibb R. Medical rapid prototyping technologies: state of the art and current limitations for application in oral and maxillofacial surgery. Journal of oral and maxillofacial surgery $\mathrm{J}$ Oral Maxillofac Surg. 2005;63(7):1006-15.

12. Muller A, Krishnan KG, Uhl E, Mast G. The application of rapid prototyping techniques in cranial reconstruction and preoperative planning in neurosurgery. J Craniofac Surg. 2003;14(6):899-914.

13. Markl M, Schumacher R, Kuffer J, Bley TA, Hennig J. Rapid vessel prototyping: vascular modeling using $3 t$ magnetic resonance angiography and rapid prototyping technology. MAGMA. 2005;18(6):288-92.

14. Wurm G, Tomancok B, Pogady P, Holl K, Trenkler J. Cerebrovascular stereolithographic biomodeling for aneurysm surgery. J Neurosurg. 2004;100(1):139-45.

15. Kimura T, Morita A, Nishimura K, Aiyama H, Itoh H, Fukaya S, Sora S, Ochiai C. Simulation of and training for cerebral aneurysm clipping with 3-dimensional models. Neurosurgery. 2009;65(4):71925.

16. D'Urso PS, Thompson RG, Atkinson RL, Weidmann MJ, Redmond MJ, Hall BI, Jeavons SJ, Benson MD, Earwaker WJ. Cerebrovascular biomodelling: a technical note. Surg Neurol. 1999;52(5):490-500.

17. Vega C, Kwoon JV, Lavine SD. Intracranial aneurysms: current evidence and clinical practice. Am Fam Physician. 2002;66(4):6018.

18. Mankovich NJ, Cheeseman AM, Stoker NG. The display of threedimensional anatomy with stereolithographic models. J Digit 
Rapid prototyping of three-dimensional biomodels as an adjuvant in the surgical planning for intracranial aneurysms

Imaging. 1990;3(3):200-3.

19. Choudhari KA, Ramachandran MS, McCarron MO, Kaliaperumal C. Aneurysms unsuitable for endovascular intervention: surgical outcome and management challenges over a 5-year period following International Subarachnoid Haemorrhage Trial (ISAT). Clin Neurol Neurosurg. 2007;109(10):868-75.

20. Wurm G, Lehner M, Tomancok B, Kleiser R, Nussbaumer K. Cerebrovascular biomodeling for aneurysm surgery: simulationbased training by means of rapid prototyping technologies. Surg Innov. 2011;18(3):294-306.
Bruna Olandoski Erbano

Rua Itupava, $240 / 3^{\circ}$ andar

80.045-140 Curitiba - PR Brasil

Tel.: (55 41)9961-4471

bruoe@hotmail.com

Received: July 18, 2013

Review: Sept 17, 2013

Accepted: Oct 21, 2013

Conflict of interest: none

Financial source: Scientific Scholarship of the Brazilian National Council of Scientific and Technology Development (PIBIC-CNPq)

${ }^{1}$ Research performed at Postgraduate Program in Principles of Surgery, Evangelic Faculty of Parana, University Evangelic Hospital of Curitiba, Medical Research Institute, Curitiba-PR, Brazil.

\section{Correspondence:}

